\title{
Contextualizing data on a Content Management System
}

\author{
Cátia Moreira \\ c.moreira@campus.fct.unl.pt \\ João Taborda \\ j.taborda@campus.fct.unl.pt \\ NOVA-LINCS, Faculdade de Ciências e Tecnologia, Universidade Nova de Lisboa \\ Campus de Caparica, 2829-516 Caparica, Portugal \\ Rosa Del Gaudio \\ Faculdade de Ciências, Departamento de \\ Informática, Universidade de Lisboa \\ Campo Grande, 1749-016 Lisboa, Portugal \\ rosa@di.fc.ul.pt \\ Lara Santos
Isantos@deetc.isel.pt \\ ISEL-Instituto Superior de Engenharia de Lisboa, Instituto Politécnico de Lisboa \\ Rua Conselheiro Emídio Navarro, 1959-007 Lisboa, Portugal
}

\begin{abstract}
Content Management Systems (CMSs) are known for their ability for storing data, both structured and non-structured data. However they are not able to associate meaning and context to the stored information. Furthermore, these systems do not meet the needs and expectations of their users, because as the size of data increases, the system loses its capacity of retrieving meaningful results.

In order to overcome this issue, we propose a method to implement data contextualization on a CMS. The proposed method consists of enriching the data with semantic information, allowing a more accurate retrieval of results. The implementation of this approach was validated by applying this contextualization method to a currently used CMS with real information. With this improved CMS, it is expected that the users will be able to retrieve data related to their initial search.
\end{abstract}

\section{Categories and Subject Descriptors}

H.3.1 [Information Storage and Retrieval]: Content Analysis and Indexing; H.3.3 [Information Storage and Retrieval]: Information Search and Retrieval

\section{General Terms \\ Information Extraction, Information Retrieval}

Permission to make digital or hard copies of all or part of this work for personal or classroom use is granted without fee provided that copies are not made or distributed for profit or commercial advantage and that copies bear this notice and the full citation on the first page. Copyrights for components of this work owned by others than ACM must be honored. Abstracting with credit is permitted. To copy otherwise, or republish, to post on servers or to redistribute to lists, requires prior specific permission and/or a fee. Request permissions from Permissions@ acm.org.

ESAIR'15, October 23, 2015, Melbourne, Australia.

(C) 2015 ACM. ISBN $978-1-4503-3790-8 / 15 / 10 \ldots 15.00$.

DOI: http://dx.doi.org/10.1145/2810133.2810134.

\section{Keywords}

Content Management System, CMS, Contextualization, Language Detection, Text Extraction, Query Expansion

\section{INTRODUCTION}

Content Management Systems (CMSs) allow the creation, editing and storage of information in content repositories. A CMS handles structured and unstructured data that is usually stored without supporting metadata or additional meaning, since they use relational databases to store data and retrieve it. Although some CMSs implement basic semantic features by exploring the metadata, they lack some important features such as semantic information that could describe the stored content [6]. The lack of semantic capabilities leads to several problems such as content duplication, the inability to correlate information and incorrect retrieval of data.

Recent advancements in Information Retrieval (IR) [7], Information Extraction (IE) [9] and Semantic Web have to be considered in the development of a new generation of CMSs. When a user needs to search through the stored data, a traditional CMS falls short of precise and meaningfully results. Even if the related content is stored in the system, the search engine may not be able to retrieve that content [2]. The search for information in these systems, that is nowadays generally based on simple text match, needs to be replaced with more thorough search methods. Semantic information and contextualization are examples of core functionalities that should exist in a CMS.

This paper describes an approach that improves a CMS by contextualizing the stored information, which consequently increases the amount of results returned by searching a term. In order to test our approach, a prototype was created using a CMS bearing real data. The prototype includes a combination of well-known techniques that were adapted to enhance the actual CMS. Although the individual techniques are not new, and references are made accordingly, the particular combination presented herein applied to a real CMS product is. 
The next sections of this paper are organized as follows. Section 2 summarizes current developments in CMSs. In Section 3, we briefly describe what contextualization is and the steps required to apply it to a CMS. Section 4 reports on the architecture of our system that was applied to a specific target CMS named Eggon Service Portal. Section 5 presents the conclusions drawn about the methods that were used.

\section{STATE OF THE ART}

The previous section emphasizes the shortcomings of the current version of the CMS, in particular the absence of semantic information associated with the stored content. To address this well-known weakness, one of the most popular techniques is the insertion of keywords that specify the content that is stored in the CMS. This technique can be applied in two ways, manually or automatically. The first one is more accurate since it relies on a human to gather the document's keywords, however this is an expensive process because it is extremely time consuming and needs the person to be knowledgeable of the document's context. The automatic method is still a challenge and resorts to techniques based on Information Extraction (IE).

The IE techniques aim at obtaining the relevant information present in the stored documents. With this information it is possible to enrich the semantic context of the documents, a feature that most CMSs lack.

In the last few years several researches have focused on improving and increasing new semantic capabilities in CMSs. In [5] an approach is proposed for browsing documents exploiting a class hierarchy of an ontology related to the content of the specific CMS. This ontology is not generated automatically, but manually. This method can help to perform a more meaningful navigation on content, however it does not enrich the content and the search with further semantically significant content.

LMF [4] allows the annotation of media content using Linked Open Data, providing storage and retrieval functionalities. The stored content and associated metadata are published using an interlinked approach.

Domain ontologies are used in [8] in order to expand the original search. The query is enriched with hypernyms, hyponyms, synonyms and related concepts.

The KIM platform [10] provides an infrastructure and different services for automatic semantic annotation, indexing, and retrieval of documents. It stores documents together with their annotations and provides various ways to search for documents. The search exploits ontologies, named entities within documents and their attributes.

\section{CONTEXTUALIZATION}

The contextualization of data is a procedure that is meant to give meaning and to add semantic information to data stored in the system. Among that data, ambiguous terms can have their significance accurately defined by exploiting information gathered from the related concepts, which will be associated with it.

Applying contextualization to a system allows the retrieval of information about a specific term and also its associated concepts. The obtained results would be significantly more reliable, because instead of just finding a match, a search applied to contextualized data obtains all the associated information and returns the results accordingly.
Information Retrieval (IR) [7] techniques can be enhanced by resorting to the inclusion of contextualization, since it will allow the existing data to be related, increasing the entanglement of information. The combination of IR with contextualization allows the data to be organized and structured, which empowers the new generation of CMSs with features that traditional ones lack.

To achieve effective data contextualization, we propose an approach that consists on determining the universe of concepts related to each text in a CMS. The goal is to create a mesh of representative concepts, which are linked together. This way, when a user performs a search we want to provide the texts where the searched expression is present, and also other texts correlated with it. The following subsections describe the necessary step to implement our idea.

\subsection{Identifying significant information}

A CMS can store different types of data from several contexts, therefore in order to contextualize the stored information, the first step is the identification of significant data. In order to perceive which information is more relevant, it is required to first conduct a domain analysis with the CMS owner. This analysis is essential for understanding the type of stored data and the fields that each system's owner valuates. With this knowledge we can adapt the techniques used for text extraction to the resources' format, the owner's requests and the domain where the contextualization is to be applied.

\subsection{Language detection and representative terms extraction}

Ideally, the contextualization should be applied to any text in any language, therefore it is required to determine the text's language. This information is valuable since each language has complex rules and different ways to build sentences. For these reasons, the text obtained from the previous step has to be submitted to a service that can detect its language. Once identified, we are able to apply a suitable keyphrase extractor.

These extractors process the text based on a list of stopwords, which contains the most common words used in a language that are not useful for identifying the context of a text. Alongside the list of stopwords, the extractors use filtering and analyzing features such as frequency statistics and occurrence positioning.

Once the text's representative terms are obtained, it is possible to identify its context, main topics and ideas. This information is useful to disambiguate terms, since the collection of topics can specify which is the text's category. Moreover, these representative terms can be used as tags to create groups of texts with similar contexts.

\subsection{Expanding the domain}

The expansion of the term's domain is obtained using the concepts directly associated to it and also the indirect associations. For instance, the term "Wine" is associated to the concepts of "Cork", "Oak" and "Grapes". Moreover, "Corkscrew" is associated to "Cork" and from this it can be inferred that "Wine" is related with "Corkscrew", and so on. These relations can be obtained using online services such as DBpedia $^{1}$ and Wikipedia ${ }^{2}$, since they allow the retrieval of

\footnotetext{
${ }^{1}$ http://wiki.dbpedia.org/

${ }^{2}$ https://en.wikipedia.org/wiki/Main_Page
} 


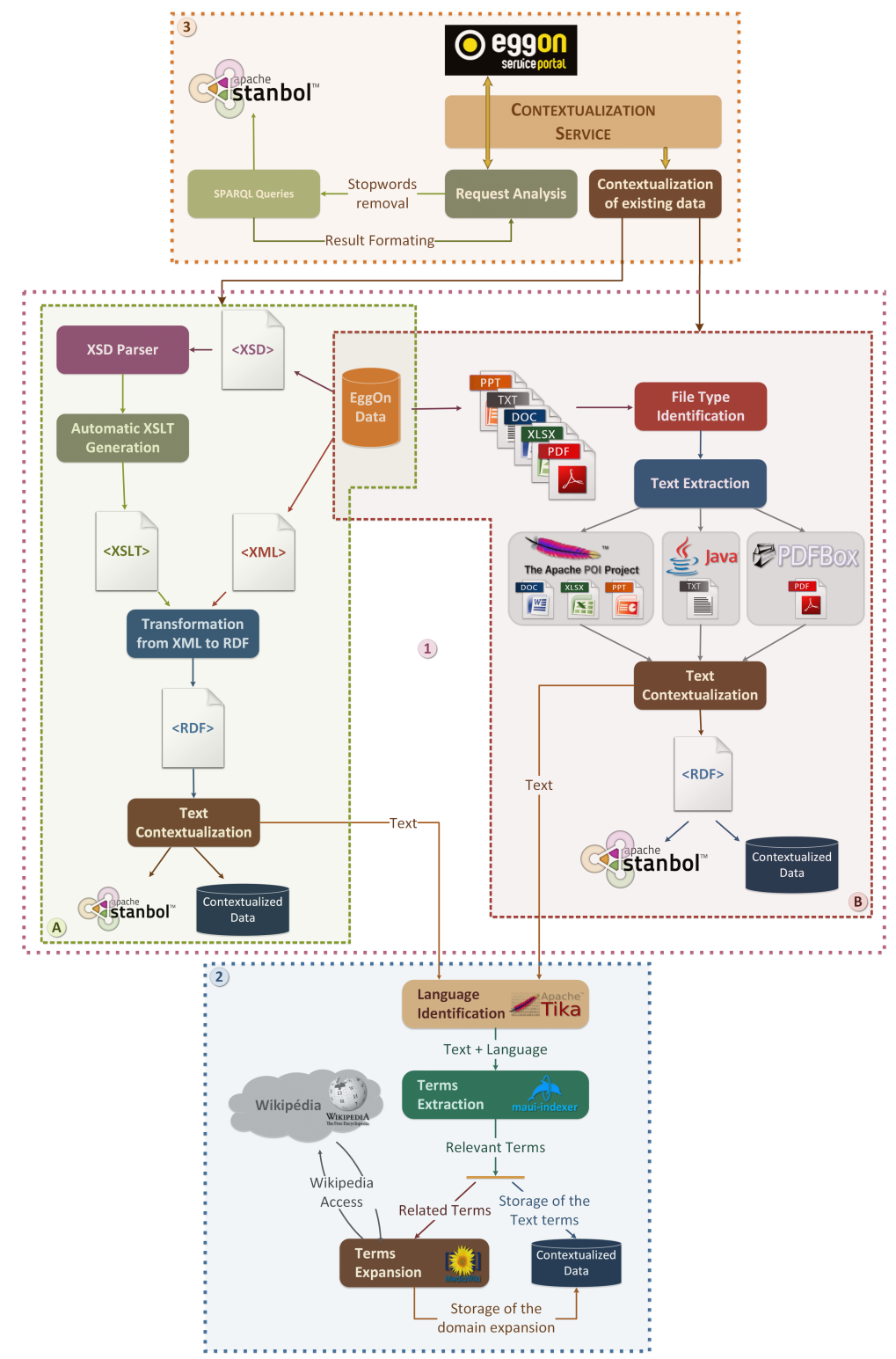

Figure 1: Contextualization of EggOn Service Portal

concepts of the same domain. This feature is required for a more precise correlation between texts with similar context.

\section{CONTEXTUALIZING THE EGGON SER- VICE PORTAL}

The previously described approach (Section 3) was implemented in a real product in order to assess the advantages of the proposed idea regarding the CMS's contextualization.

The integration of our solution was accomplished in association with the Portuguese company Flow Options ${ }^{3}$. This partnership allowed us to validate the contextualization service in their CMS, named EggOn Service Portal. The Service Portal product is a proprietary CMS that enables its users to create and manage tasks, services and processes from several departments in a company, providing storage of

\footnotetext{
$\overline{{ }^{3} \text { http://www.flowoptions.com/ }}$
}

different types of data in different file formats (XML, PDF, Word and others).

For this specific implementation of our approach, contextualization is divided into three parts: (i) Text Extraction, (ii) Contextualization Engine and (iii) Contextualization Service. The first part consists of the extraction of relevant text from the EggOn Database. The extracted text is then submitted to the Contextualization Engine, that identifies the text's language, concepts and domain. The contextualized information resulting from this module is saved in a Contextualization Database and in the Apache Stan$\mathrm{bol}^{4}$, using the RDF format. Apache Stanbol is a framework that combines a set of components for data optimization with semantic services. One of the functionalities of Apache Stanbol is the ability to perform SPARQL queries on data formatted in RDF triples. This type of queries is faster

\footnotetext{
${ }^{4}$ https://stanbol.apache.org/
} 


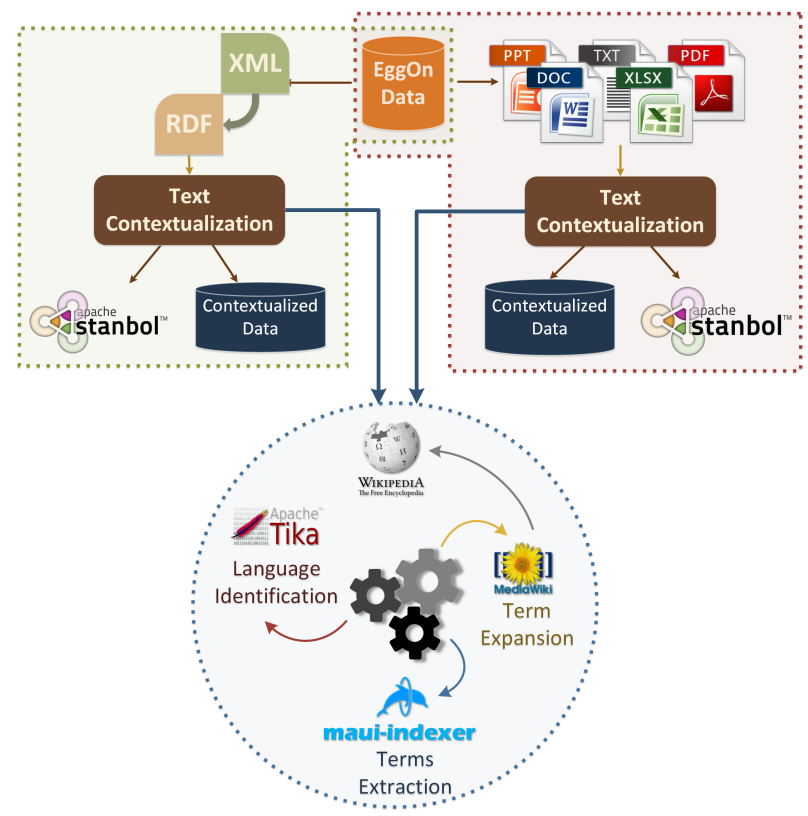

Figure 2: Text Extraction module architecture

than traditional ones, since it relies on a graph of connected nodes.

Finally, the Contextualization Service is responsible for providing the contextualized information and also for processing new data added to the EggOn Database. The described process, depicted in Figure 1, is composed by the following modules:

1. Text Extraction module

(a) XML Translation module

(b) File Processing module

2. Contextualization Engine module

3. Contextualization Service module

The last section presents some of the obtained results when performing tests with our prototype, highlighting those where more significant differences where detected when comparing the previous search functionality with the new one implemented in the EggOn Service Portal.

\subsection{Text Extraction and Data Contextualiza- tion}

The EggOn Service Portal manages two types of sources of information to be contextualized. The first type is the one regarding the forms filled by the users in the CMS interface which, upon submission, is stored using a XML format. The second type is pertaining to files (PDF, Word, Excel, etc.) that can be uploaded into the CMS. Both types of sources of information should be stripped, in order to obtain the raw text to be contextualized.

Figure 2 depicts the architecture of the module that contextualizes the significant data from the EggOn Database. Due to the fact that each type of data (files or XML) has different contexts, it was necessary to split them in two distinct sub-modules, the XML Translation (Section 4.1.1) and the File Processing (Section 4.1.2).
Both sub-modules extract the text of each document, which is afterwards submitted to the Contextualization Engine module (Section 4.2). The information obtained by the Contextualization Engine is required for tagging each document with its context and main topics.

Each sub-module produces a RDF file for each document, which arranges all the information in a triple system. Each triples[1][3] is composed of a Subject, a Predicate and an Object. The Subject is linked to an Object by a Predicate, which generates a graph of connections between all the elements involved.

\subsubsection{XML Translation}

The EggOn Service Portal supports both Issues and Service Requests that are created by users. This information is stored in a Relational Database in the form of XML, however, the schema of each XML is not the same, since it is adapted according to the information that is being represented.

With this in mind, a few steps are necessary to perform the conversion from XML to RDF, as depicted in Figure 3.

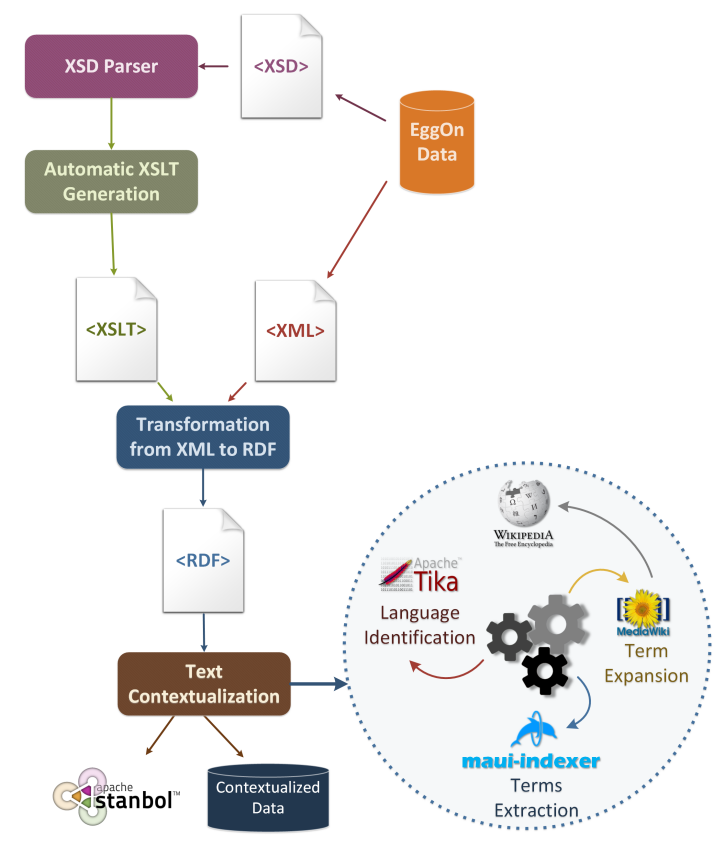

Figure 3: Process to achieve the Xml translation

The first step consists in parsing the XSD files stored in the EggOn Database, considering that these files define the structure of the stored XML encoded data. Each XSD is decomposed in order to identify the elements' structure and their relationships. Using this information, a XSLT is generated for each existing XSD, allowing the XML to be transformed in RDF triples. This transformation is a fundamental part in this approach, because it organizes the information in the form of triples, which consequently, allows the building of a graph representing all the established relationships between each processed document.

After a successful conversion from XML to RDF, the RDF files' texts are submitted to the Contextualization Engine (Section 4.2), to retrieve the keyphrases that identify each XML. The execution of this module ends when each RDF is 
stored in Apache Stanbol, containing the initial information and the collected tags.

\subsubsection{File processing}

In addition to XML files, the EggOn Database also supports the storage of other types of files that also require contextualization. Since the stored files could have several types of extensions, the aim of this module is to handle the different ways of extracting text from them. An overview of the process used in this module can be seen in Figure 4.

The first phase is the identification of the extensions extracted from the files stored in the database. Once identified, the file is redirected to a specific library that is capable of handling that particular file format. This library's target is the text extraction that is then sent to the Contextualization Engine (Section 4.2) to extract the representative concepts of the submitted texts. The produced RDF file contains information about the file such as its name, extension and related concepts.

On this first version, it would be uncalled-for the system to be handling all the possible file extensions that could contain text. For this reason, we only target the types of files that are currently supported by the Service Portal, namely:

- Microsoft Office extensions (.doc, .docx, .xls, .xlsx, .ppt, .pptx)

The handling of these files is performed by resorting to the Apache $\mathrm{POI}^{5}$ library. This library has several functionalities, including the extraction of specific sections of a file (body, header, footer, captions) and also the file's metadata.

- PDF files (.pdf)

The PDF format is widely used since its main feature is to maintain the file's aspect between devices. To handle this format, the Apache PDFBox ${ }^{6}$ library was used. This library offers the functionality of extracting the file's content.

- Text files without format (.txt)

To read raw text from files, we do not need any library in particular, since the native functionalities of programming platforms in general (like Java in our case) offer the possibility to handle this type of files directly.

We chose to leave aside the files with image extensions, like .jpeg, png or .bmp, since the techniques from the extraction of words for those formats are not yet full-proof, resulting in inaccurate texts.

\subsection{Contextualization Engine}

The Contextualization Engine procedure described in this section is composed by three phases, which are detailed in Figure 5. This module receives raw text that is parsed differently in each phase, aiming for the extraction of useful information to generate contextualized data.

This module can be considered as the core of our project, since it enhances the search functionality by improving the amount of results obtained. This is possible because the search is based on a collection of topics associated with a text, instead of considering a search in the text itself.

\footnotetext{
${ }^{5}$ https://poi.apache.org/

${ }^{6}$ https://pdfbox.apache.org/
}

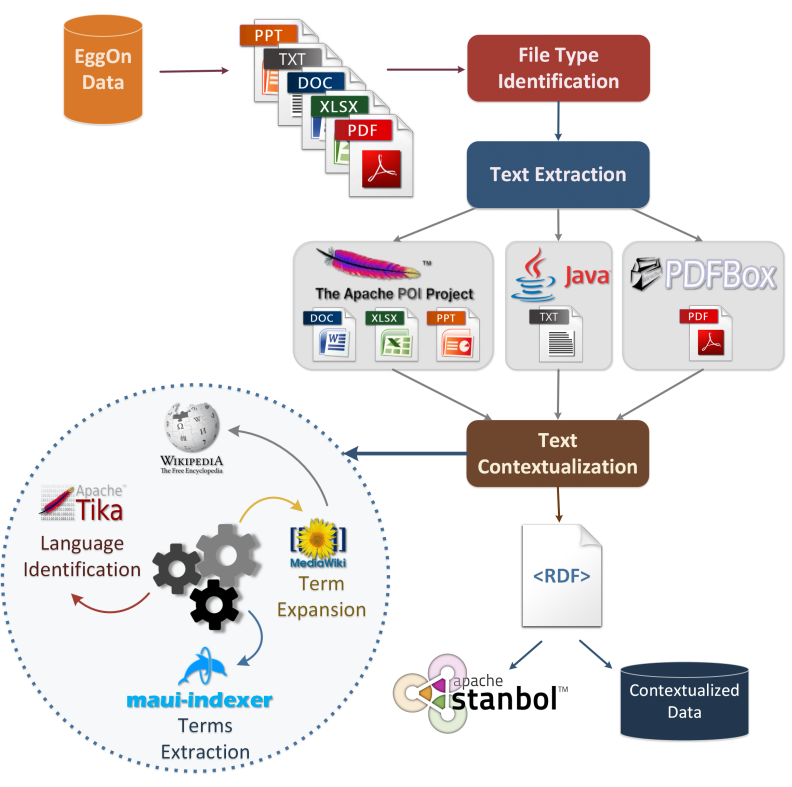

Figure 4: Process for File parsing

The following subsections describe each of the three phases mentioned above which are required to perform contextualization.

\subsubsection{Analysis}

The raw texts received can have two distinct sources, they can be extracted from the XML files processed by the module described in Section 4.1.1 or from the text files that were submitted to the module presented in Section 4.1.2.

The analysis phase intends to determine the text native language, because in the next phase (Section 4.2.2) this information is crucial in order to obtain more knowledge about the topics extracted.

Language detection is performed by using Apache Tika ${ }^{7}$. This toolkit can detect and extract metadata from texts, using the same interface. Its features include indexing, content analysis, translation and many others, which makes this toolkit an appealing solution to detect the language and to expand the prototype with other functionalities in the future.

\subsubsection{Extraction}

Once the source language is identified, there is the need to collect the more relevant terms from the text. This procedure, conducted in this phase, allows the extraction of a relevant set of topics that could fit into a category. To perform the extraction, we used maui-indexer, ${ }^{8}$ a tool that automatically identifies the main topics of a given text in any language, using a specific file of stopwords to be ignored. Currently, the prototype supports both Portuguese and English texts, but it is prepared to be extended by adding a file with the stopwords for another language.

Even with the main topics identified, we have not collected the necessary concepts to build a contextualized domain, so we must search deeper for more terms that are related to the extracted ones.

\footnotetext{
${ }^{7}$ https://tika.apache.org/

${ }^{8}$ https://github.com/zelandiya/maui
} 


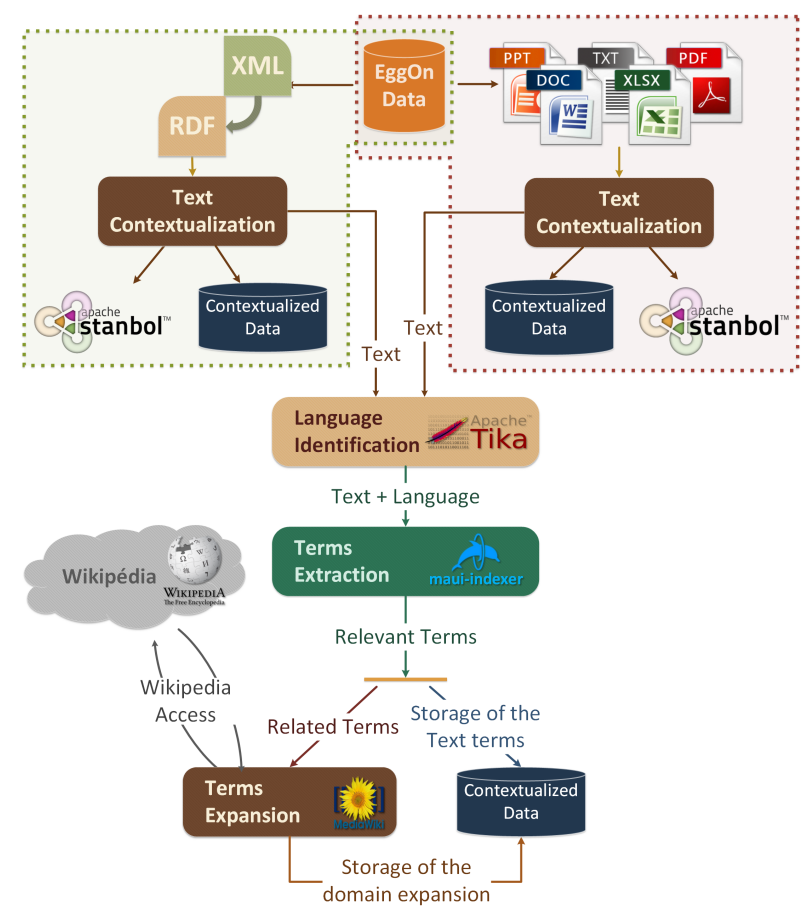

Figure 5: Scheme of the Contextualization Engine module

\subsubsection{Domain Expansion}

The last phase of the developed prototype has the purpose of building a mesh of terms related to the originally searched term. In order to optimize the search of the documents where the term (and all the others related to it) is present, all the texts are tagged with the relevant words extracted. This operation makes the search action effective, since we do not need to process the text again.

To build this network of terms and related concepts we need a source of information. For testing purposes, we decided to use Wikipedia ${ }^{9}$ because it is a free online encyclopedia in constant growth. The Wikimedia Foundation ${ }^{10}$ has many other projects alongside Wikipedia, being one of them the Media $\mathrm{Wiki}^{11}$ package. Using its functionalities, we can obtain the Wikipedia's pages based on a term, which allows the extraction of more concepts that will be used to build the network of terms.

\subsection{Contextualization Service}

The Contextualization Service is responsible for the communication between the EggOn Service Portal and the other modules described in the previous sections. This module is based on a Web Service which sends and receives information. This service also defines a Scheduler that updates the Contextualization Database with the contextualized data, as shown in Figure 6. This module is executed according t a configuration file which specifies the databases to be accessed, the ports where the service should run and also the time interval to schedule the retrieving of new data.

\footnotetext{
${ }^{9}$ https://en.wikipedia.org/wiki/Main_Page/

${ }^{10}$ https://en.wikipedia.org/wiki/Wikimedia_Foundation

${ }^{11}$ https://www.mediawiki.org/wiki/MediaWiki
}

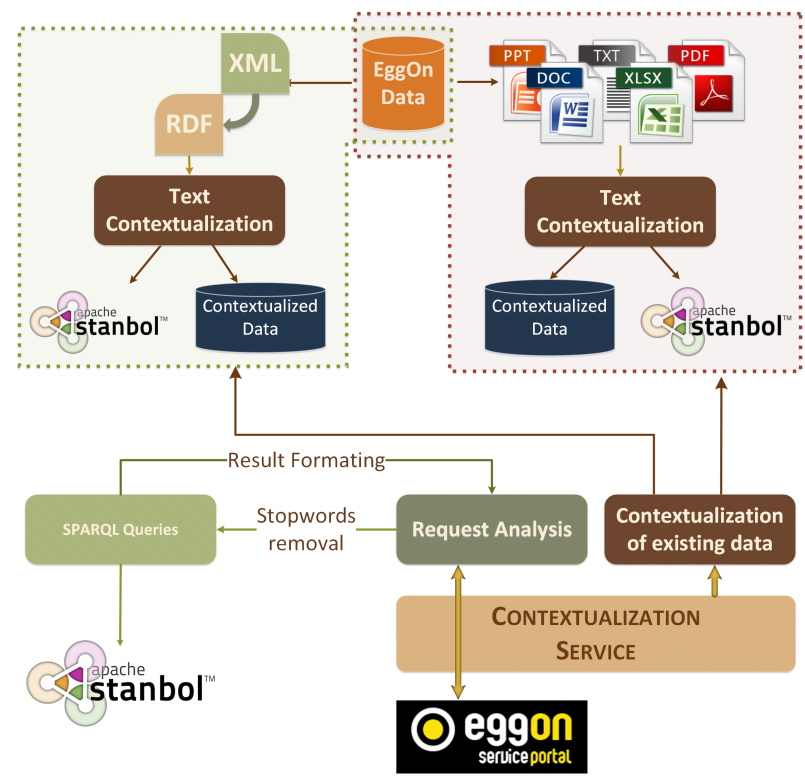

Figure 6: Scheme of the Contextualization Service module

\subsubsection{Contextualization Scheduling}

The EggOn Service Portal is a system of everyday use, which consequently leads to a constant growth of data. Moreover, since this system is working independently, our integration point was a scheduling service that periodically reads the new inserted data. This service is executed based on the time interval defined in the configuration file and performs both the XML Translation (Section 4.1.1) module and the Files Processing (Section 4.1.2) module.

\subsubsection{Web Service}

The Contextualization Service also initiates a Web Service that returns the data associated with the specific search. This search is performed by removing the stopwords, using the same techniques used in the Contextualization Engine (Section 4.2). For each term extracted from the XML and the files stored in the EggOn database, a SPARQL query is performed to obtain the results that belong to the search domain. The final result is formatted into JSON and includes the database entry ID and an excerpt from the text where the term is present.

\subsection{Contextualization Validation}

The first version of the prototype was implemented in the EggOn Service Portal and tested with real users to compare the evolution of the search functionality.

Throughout the test phase, a repetition of three distinct patterns stood out. To explain in more detail these patterns, we chose three concrete searches that represent each one of them. This section also describes the metrics used and the discussion of the obtained results.

\subsubsection{Validation Process}

The efficiency of the Contextualization Engine was validated by performing several tests over the existing entries of the EggOn Database. These tests were conducted by comparing the search results obtained in three distinct types of search: (i) Simple, which is based on word-matching, in 
other words, the direct search of the term in the text; (ii) Related, where the search is based on the relevant terms extracted from each document and (iii) Contextualized, the approach described in this paper. In the latter, the search is performed based on the expansion of the extracted relevant terms.

For each one of these search types, we used performance metrics to determine the precision, the recall and the f-measure of the collection of elements [7][9].

The precision allows to determine the percentage of correct results. The recall calculates the percentage of correct results obtained after the search. Using both the recall and precision values, it is possible to determine the f-measure, which is a weighted harmonic mean between them. The $\mathrm{f}$-measure indicates the search efficiency $\mathrm{v}$, therefore the $\beta$ value is 1 . To calculate each one of these three values, the following formulas were used:

$$
\begin{aligned}
\text { precision } & =\frac{\# \text { correct }}{\# \text { correct }+\# \text { incorrect }} \\
\text { recall } & =\frac{\# \text { correct }}{\# \text { expectedResults }} \\
f-\text { measure } & =\frac{\left(\beta^{2}+1\right) * \text { precision } * \text { recall }}{\left(\beta^{2} * \text { precision }\right)+\text { recall }}
\end{aligned}
$$

\subsubsection{Results Analysis}

The procedure consisted in searching several possible terms using the three types of search. As an example, we present the following terms: Residence, Energy Storage and Urban Renewal. These terms were selected because they illustrate the different aspects in each search type. The table 1 displays the data used for the previously described metrics.

\section{Residence}

As illustrated in Figure 7, the results obtained for the term "Residence" show that both the Simple Search and the Contextualized Search have $100 \%$ of precision, since all the returned results were considered relevant. However, Related Search did not produced any value, due to the fact that the term "Resident" was considered irrelevant during the keyword extraction of the texts.

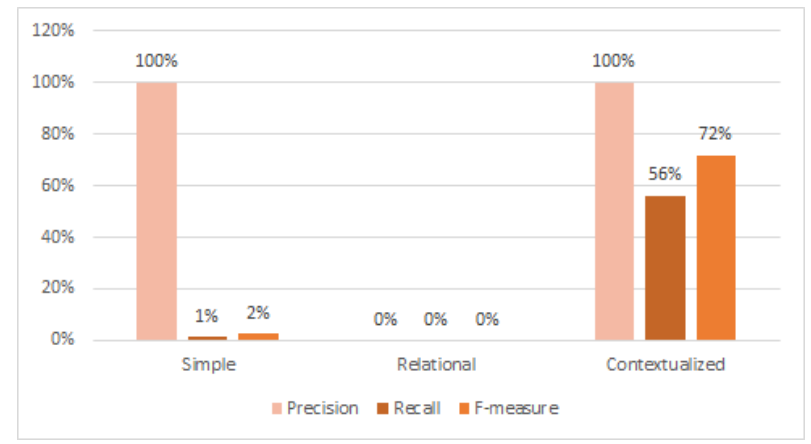

Figure 7: The metrics calculated for the term "Residence"

Comparing the recall values obtained in the Simple Search and in the Contextualized Search, we can observe that the latter is able to retrieve more of the expected results. This type of search obtains more terms than just a simple wordmatching, by fetching the topics that are related to the "Residence" universe.

Considering that both of these values have the same weight ( $\beta$ value), the f-measure can be calculated. Observing Figure 7 , we can see that the Contextualized Search has an efficiency level of $72 \%$.

\section{ENERGy STORAGE}

For this case (Figure 8), the Simple Search is unable to return correct results, since the expression "Energy Storage" does not exist in any document stored in the EggOn Database.

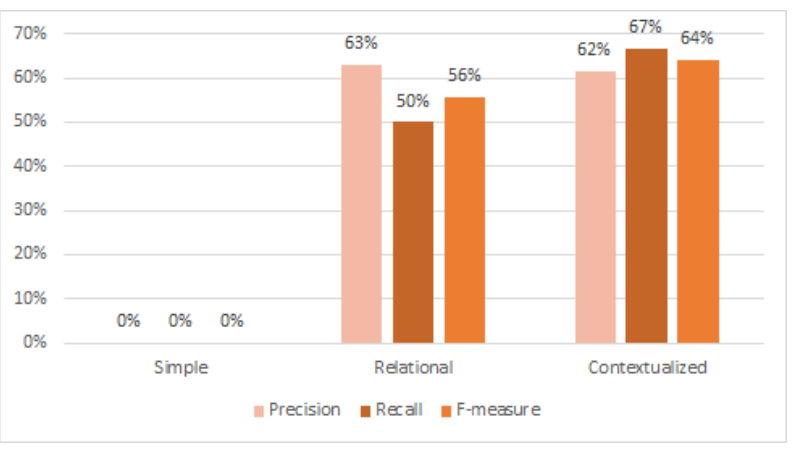

Figure 8: The metrics calculated for the term "Energy Storage"

Comparing the precision of the Related Search with the Contextualized Search, the Related one has more advantage since the amount of results obtained is smaller. However, the Contextualized Search outranks the others with a recall of $67 \%$, resulting in an efficiency level of $64 \%$.

\section{URBAN RENEWAL}

This last example presents a case where the Contextualized Search do not cover more than half of the results, as can be seen on the Figure 9, resulting in a recall value of $44 \%$. This case is representative of one of the weaknesses of our prototype, because the Contextualized Search returns several incorrect results, which leads to a low precision and low recall, consequently leading to an efficiency level of $41 \%$.

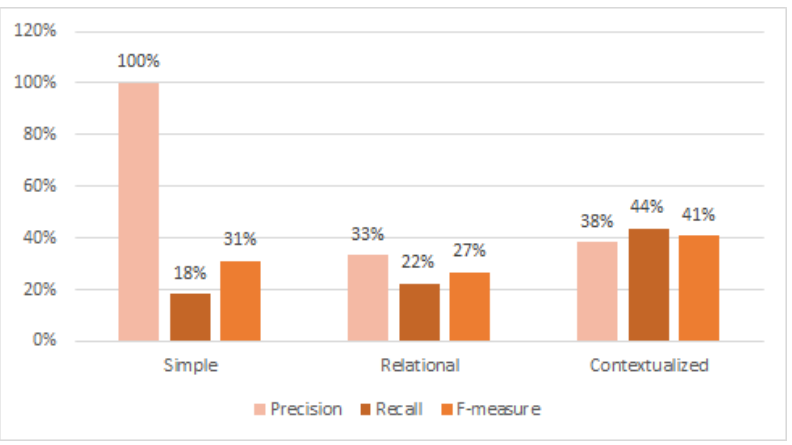

Figure 9: The metrics calculated for the term "Urban Renewal" 
Table 1: Validation Results Sample

\begin{tabular}{|c|c|c|c|c|c|c|c|c|}
\hline $\begin{array}{c}\text { Searched } \\
\text { Term }\end{array}$ & $\begin{array}{c}\text { Search } \\
\text { Type }\end{array}$ & $\begin{array}{c}\text { \#Expected } \\
\text { Results }\end{array}$ & $\begin{array}{c}\text { \#Correct } \\
\text { Results }\end{array}$ & $\begin{array}{c}\text { \#Missing } \\
\text { Results }\end{array}$ & $\begin{array}{c}\text { \#Incorrect } \\
\text { Results }\end{array}$ & Precision & Recall & f-mesaure \\
\hline \multirow{3}{*}{ Residence } & Simple & \multirow{3}{*}{637} & 8 & 0 & 629 & 1 & 0.01 & 0.02 \\
\hline & Related & & 0 & 0 & 637 & 0 & 0 & 0 \\
\hline & Contextualized & & 358 & 0 & 279 & 1 & 0.56 & 0.72 \\
\hline \multirow{3}{*}{$\begin{array}{l}\text { Energy } \\
\text { Storage }\end{array}$} & Simple & \multirow{3}{*}{34} & 0 & 0 & 34 & 0 & 0 & 0 \\
\hline & Related & & 17 & 10 & 17 & 0.63 & 0.50 & 0.56 \\
\hline & Contextualized & & 32 & 20 & 16 & 0.62 & 0.67 & 0.64 \\
\hline \multirow{3}{*}{$\begin{array}{c}\text { Urban } \\
\text { Renewal }\end{array}$} & Simple & \multirow{3}{*}{197} & 37 & 0 & 165 & 1 & 0.18 & 0.31 \\
\hline & Related & & 42 & 84 & 145 & 0.33 & 0.22 & 0.27 \\
\hline & Contextualized & & 86 & 139 & 110 & 0.38 & 0.44 & 0.41 \\
\hline
\end{tabular}

\subsubsection{Results Discussion}

The first version of the Contextualization Search was compared with the two search types (Simple and Related), one that is based in word-matching and the other on the most relevant words present in a text.

The comparison made in the previous section led us to some important conclusions described below.

The Simple Search can reach higher values of precision, but on the other hand, it is unable to cover a high percentage of the expected terms. This flaw comes from the simple word-matching search performed, resulting in both really high or really low values of precision. This type of search is undesirable, because it does not consider terms and topics related to the original one.

The Related Search is based on the terms previously extracted from the documents stored in the database. This fact makes the precision for this type of search inaccurate, because if the searched term is not considered relevant by the Keywords Extractor, the file document is not contemplated. Additionally, the same term can be used in documents with distinct context, resulting in more incorrect results, and consequently in various recall values.

Finally, the Contextualized Search did not performed as bad as the Related Search, but it is far from being perfect, since it suffers from the same problem as the previous. In spite of this issue, this type of search consistently returns a larger amount of results than the other ones and is successful at covering most of the documents in the database. This fact results in higher recall values, as this search is not only based on the extracted terms, but also in the concepts related with them.

Although the Contextualized Search has proven to have levels of efficiency between $40 \%$ and $75 \%$ there are still margin for improvement. The reduction of incorrect results is one of the problems to focus on for achieving higher precision values, which will result in more relevant documents obtained. Besides improving the precision values, the percentage of recall should be over $80 \%$, because we need a system that is capable of returning the majority of the documents stored. This can be accomplished by optimizing the Extraction phase (Section 4.2.2) and the Domain Expansion phase (Section 4.2.3). The Extraction phase has to be able to retrieve more terms from each document, conducting a more thorough selection of the main topics of a text. The Domain Expansion phase should include more online services to expand the network of related concepts of a term. Alongside these improvements, it is also important to support the search for different languages using term translation for the next version of the prototype.

\section{CONCLUSIONS}

This paper describes our approach to contextualization and how it can be applied to a CMS. To summarize, we first need to identify the data to be contextualized and extract the contained text and corresponding language. The next step consists of the extraction of relevant terms and finally, the execution of a deep search online for retrieving more concepts from each term's domain.

The actual state of the prototype has proven to gather better results when compared to the previous search engine. Before the prototype, the search functionality was limited to word-matching, which returned basic results that were not relevant to the users of EggOn Service Portal. This feature was enhanced, but as shown on the conducted tests, there is still room for improvement. The Contextualized Search is not capable of covering more than $80 \%$ of the relevant results present in the database and returns more irrelevant documents than desirable.

After the prototype revision with the implementation of these enhancements, we intend to include other functionalities. The search action could offer the possibility of searching through a certain level of deepness, which can be defined by the user. Furthermore, the user can specify if he wants to limit his search to certain categories that only exist in the collection of data present in the CMS. This feature can be even more expanded by creating an adaptive system that returns only results of interest for that specific user.

\section{ACKNOWLEDGMENTS}

The authors of this paper thank QREN Project number FO13_IDTEggon for the financial support and also the Flow Options company for providing the resources to implement and test the prototype.

\section{REFERENCES}

[1] J. J. Carroll and P. Stickler. RDF Triples in XML. In Proceedings of the 13th International World Wide Web Conference on Alternate Track Papers EAmp; Posters, WWW Alt. '04, pages 412-413, New York, NY, USA, 2004. ACM.

[2] M. R. Ghorab, D. Zhou, A. O'Connor, and V. Wade. Personalised Information Retrieval: survey and classification. User Modeling and User-Adapted Interaction, 23(4):381-443, May 2012.

[3] G. Klyne and J. J. Carroll. Resource Description Framework (RDF): Concepts and Abstract Syntax. Technical report. 
[4] T. Kurz, S. Schaffert, and T. Burger. Lmf: A framework for linked media. Multimedia on the Web, Workshop on, 0:16-20, 2011.

[5] G. Laleci, G. Aluc, A. Dogac, A. A. Sinaci, O. Kilic, and F. Tuncer. A semantic backend for content management systems. Knowl.-Based Syst., 23(8):832-843, 2010.

[6] W. Maass and T. Kowastsch. Semantic Technologies in Content Management Systems. 2012.

[7] C. D. Manning, P. Raghavan, and H. Schütze. Introduction to Information Retrieval. Cambridge University Press, New York, NY, USA, 2008.

[8] W. A. Pinheiro and A. M. de Carvalho Moura. An ontology based-approach for semantic search in portals. In DEXA Workshops, pages 127-131. IEEE Computer Society, 2004.

[9] J. Piskorski and R. Yangarber. Information extraction: Past, present and future. In T. Poibeau, H. Saggion, J. Piskorski, and R. Yangarber, editors, Multi-source, Multilingual Information Extraction and

Summarization, Theory and Applications of Natural Language Processing, pages 23-49. Springer Berlin Heidelberg, 2013.

[10] B. Popov, A. Kiryakov, A. Kirilov, D. Manov, and O. M. Goranov. Semantic annotation platform. In In Proc. of the 2 nd Intl. Semantic Web Conf. 2003, pages 834-849. Springer, 2003. 